ten zunächst unterdosieren, wegen der medizinischen und sozialmedizinischen Risiken eines großen Anfalls ist das ein wirkliches Dilemma. An medikamentöse Therapiealternativen sollte frühzeitig gedacht werden. Die eingeschränkte Zulassungssituation bei idiopathischen generalisierten Epilepsien wird die Epileptologen rasch dahin bringen, Substanzen außerhalb ihrer Zulassung in Erwägung ziehen zu müssen. Ob wir es aber verantworten können, Mädchen und junge Frauen früh mit niedrig dosierter Valproin- säure zu behandeln, bleibt für mich eine ungelöste Frage. Einzelfallentscheidungen und ein schwieriges Abwägen bleiben uns da nicht erspart. Dr. med. Martin Finzel

1. Rote Hand Brief: Arzneimittel, die Valproat und - verwandte Substanzen enthalten: Risiko für Anomalien des Neugeborenen. AKDÄ 12/2014

2. Krämer G. "Time to say goodbye" oder was wird aus der guten alten Valproinsäure bei weiblichen Jugendlichen und prämenopausalen Frauen? Akt Neurol 2015; 42:6-7

\title{
Epilepsie und Schwangerschaft - nicht nur Valproinsäure ist riskant
}

\section{Der Einsatz von antiepileptischen Polytherapien unter Einschluss von Topiramat in der Schwangerschaft ist kritisch zu sehen - neben kognitiven Entwicklungsstörungen sollte auch das Teratogenitätsrisiko beachtet werden.}

n der jüngeren Vergangenheit erschienen zahlreiche Arbeiten zum Fehlbildungspotential einzelner antiepileptischer Substanzen, insbesondere auch hinsichtlich kognitiver Entwicklungsstörungen nach fetaler ValproinsäureExposition, die Anzahl an Publikationen zum Teratogenitätsrisiko von antiepileptischen Polytherapien hingegen ist eher gering. Dies ist wahrscheinlich der großen Anzahl von pharmakologischen Kombinationsmöglichkeiten geschuldet.

Bei klarer Datenlage für eine unter Polytherapie gegenüber Monotherapie erhöhte Teratogenität wurde in der Vergangenheit bereits mehrfach gezeigt, dass nicht unbedingt die Behandlung mit zwei Medikamenten an sich einen kritischen Faktor darstellen muss, sondern dass insbesondere der Einsatz von Valproat in Kombination das Fehlbildungsrisiko erhöht. Angesichts der mittlerweile rückläufigen Verschreibung von Valproat bei Frauen im gebärfähigen Alter interessierten sich die Studienautoren des Universitätsklinikums Melbourne für die Fehlbildungshäufigkeit unter antiepileptischen Kombinationstherapien unter Ausschluss dieser als hoch teratogen eingeschätzten Substanz.

Grundlage der Studie ist eine datenbankbasierte Auswertung von Schwangerschaften (Australian Antiepileptic
Drugs in Pregnancy Register) im Zeitraum 1999 bis 2014. Bei 1.461 Schwangerschaften erfolgte eine Monotherapie, bei 484 eine Kombinationsbehandlung. Erwartungsgemäß lag die globale Fehlbildungsrate unter Polytherapie signifikant höher als unter Monotherapie $(8,06 \%$ vs. $5,48 \%$; OR 1,51; $95 \%$-KI 1,02-2,25). Noch deutlicher zeigte sich dieses Ergebnis nach Ausschluss von Valproat-exponierten Schwangerschaften $(6,90 \%$ vs. $3,64 \%$; OR 1,96; $95 \%$-KI 1,14-3,39). In der Längsschnittbetrachtung kam es zu einer Abnahme der Fehlbildungen bei Monotherapieschwangerschaften. Erstaunlicherweise wurde hingegen im Beobachtungszeitraum eine Zunahme des Risikos unter Polytherapien trotz stetig sinkender Valproat-Verschreibungszahlen und -dosierungen beobachtet.

Die Daten zeigen eine Beschleunigung dieses Anstiegs ab etwa 2005, also etwa zwei Jahre nachdem die Verordnungszahlen für Levetiracetam (LEV) und Topiramat (TPM) deutlich zugenommen hatten. Dabei unterscheiden sich die Malformationsraten bei Kombinationstherapien unter Einschluss von LEV nicht klar von solchen ohne dieses Medikament $(7,14 \%$ vs. $8,38 \%)$, wohingegen sie beim Add-on-Einsatz von TPM signifikant erhöht waren $(14,94 \%$ vs. 6,55\%). Logistische Regressionsanalysen
3. Elger CE et al. Vorsicht bei Valproat für Frauen. Akt Neurol 2015; 42: 8-10

4. Baker GA et al. IQ at 6 years after in utero exposure to antiepileptic drugs. A controlled cohort study. Neurology 2015; 84: 382-90

5. Bromley RL et al. Cognition in school-aged children exposed to levetiracetam, topiramate, or sodium valproate. Neurology 2016; 87: 1 - 11

6. Hernández-Vanegas LE et al. High-dose vs. low-dose valproate for the treatment of juvenile myoclonic epilepsy: Going from low to high. Epilepsy Behav 2016; 61: 34-40 zeigen zudem eine dosisabhängige Risikozunahme für eine TPM-Exposition in der Kombinationsbehandlung. Die Ergebnisse legen nach Meinung der Autoren trotz niedriger Fallzahlen (48 TPM-Monotherapie, 87 TPM-Polytherapie) nahe, dass der Einsatz von TPM in Polytherapien dessen teratogene Effekte verstärken könnte.

Neben den geringen Fallzahlen ist kritisch anzumerken, dass beim Einsatz von TPM in Monotherapie im Vergleich zu Schwangerschaften ohne Antiepileptika-Exposition keine erhöhten Fehlbildungsraten registriert wurden (TPM: 2,08\%; ohne Antiepileptikum 3,14\%), was von den Autoren auch eingeräumt wird. Darüber hinaus erhielten die mit TPM in Kombinationstherapie behandelten Frauen auch eine höhere Dosierung (25 - $700 \mathrm{mg} / \mathrm{Tag}, \emptyset 247,7 \mathrm{mg} / \mathrm{Tag}$;) als in Monotherapie $(6,25-400 \mathrm{mg} / \mathrm{Tag}$, $\emptyset 186,9 \mathrm{mg} / \mathrm{Tag})$.

\section{Kommentar}

Die Studie sollte Anlass dazu geben, den Einsatz von antiepileptischen Polytherapien unter Einschluss von TPM in der Schwangerschaft kritisch zu sehen, zumal sich bereits in einer früheren Veröffentlichung aus dem britischen Schwangerschaftsregister ungünstige Daten für dieses Medikament in Kombinationstherapien ergeben hatten [2].

Dr. med. Peter Hopp

1. Vaja FJ et al. Antiepileptic drug combinations not involving valproate and the risk of fetal malformations. Epilepsia 2016; 57 (7): 1048-52

2. Hunt $\mathrm{S}$ et al. Topiramate in pregnancy: Preliminary experience from the UK Epilepsy and Pregnancy Register. Neurology 2008; 71: 272-6 